\title{
Bulbar Acetylcholine Enhances Neural and Perceptual Odor Discrimination
}

\author{
Dipesh Chaudhury, ${ }^{\star}$ Olga Escanilla, ${ }^{*}$ and Christiane Linster \\ Department of Neurobiology and Behavior, Cornell University, Ithaca, New York 14850
}

\begin{abstract}
Experimental and modeling data suggest that the circuitry of the main olfactory bulb (OB) plays a critical role in olfactory discrimination. Processing of such information arises from the interaction between $\mathrm{OB}$ output neurons local interneurons, as well as interactions between the $\mathrm{OB}$ network and centrifugal inputs. Cholinergic input to the $\mathrm{OB}$ in particular has been hypothesized to regulate mitral cell odorants receptive fields (ORFs) and behavioral discrimination of similar odorants. We recorded from individual mitral cells in the $\mathrm{OB}$ in anesthetized rats to determine the degree of overlap in ORFs of individual mitral cells after exposure to odorant stimuli. Increasing the efficacy of the cholinergic neurotransmission in the OB by addition of the anticholinesterase drug neostigmine $(20 \mathrm{mM})$ sharpened the ORF responses of mitral cells. Furthermore, coaddition of either the nicotinic antagonist methyllycaconitine citrate hydrate (MLA) (20 mM) or muscarinic antagonist scopolamine $(40 \mathrm{~mm})$ together with neostigmine $(20 \mathrm{~mm})$ attenuated the neostigmine-dependent sharpening of ORFs. These electrophysiological findings are predictive of accompanying behavioral experiments in which cholinergic modulation was manipulated by direct infusion of neostigmine, MLA, and scopolamine into the OB during olfactory behavioral tasks. Increasing the efficacy of cholinergic action in the $\mathrm{OB}$ increased perceptual discrimination of odorants in these experiments, whereas blockade of nicotinic or muscarinic receptors decreased perceptual discrimination. These experiments show that behavioral discrimination is modulated in a manner predicted by the changes in mitral cell ORFs by cholinergic drugs. These results together present a first direct comparison between neural and perceptual effects of a bulbar neuromodulator.
\end{abstract}

Key words: olfactory; acetylcholine; ACh; basal forebrain; behavior; neuromodulation; receptive field

\section{Introduction}

Cholinergic inputs to cortical processing networks have long been proposed to be associated with attentional and top-down processing (Hasselmo et al., 1992; Sarter and Bruno, 1997; Sarter et al., 2005; Yu and Dayan, 2005; Hasselmo and Giocomo, 2006). In primary sensory areas, several studies have associated acetylcholine with contrast modulation and changes in neuronal receptive fields as well as the plasticity of receptive fields (Metherate et al., 1988; Metherate and Weinberger, 1990; Wilson, 2001; Weinberger, 2003; Roberts et al., 2005; Timofeeva et al., 2005). Because of the strong correlations between sensory inputs, neural activity, and perception, the transformation of sensory perception by cholinergic inputs is directly measurable in the olfactory bulb $(\mathrm{OB})$ of rodents (Linster et al., 2001b; Cleland et al., 2002, 2007; Cleland and Linster, 2005). Experimental and modeling data indicate that the main $\mathrm{OB}(\mathrm{MOB})$ plays a critical role in feature extraction, noise reduction, and contrast enhancement of odor-evoked sensory information (for review, see Cleland and Linster, 2005). A crucial function of the $\mathrm{OB}$ is to integrate afferent information

\footnotetext{
Received Aug. 24, 2008; revised Nov. 5, 2008; accepted Nov. 24, 2008.

This work was supported by National Institutes of Health Grant 1R01DC009948-01 (Collaborative Research in Computational Neuroscience). We thank Zach Nichols for discussions about data analysis.

${ }^{*} D$.C. and 0. .E. contributed equally to this work.

Correspondence should be addressed to Christiane Linster, Neurobiology and Behavior, W257 Seeley G. Mudd Hall, Cornell University, Ithaca, NY 14853. E-mail: CL243@cornell.edu.

DOI:10.1523/JNEUROSCI.4036-08.2009

Copyright $\odot 2009$ Society for Neuroscience $\quad$ 0270-6474/09/290052-09\$15.00/0
}

from the sensory neurons in concert with descending cortical and neuromodulatory inputs from central structures such as the piriform cortex, basal forebrain, and brainstem (Halász and Shepherd, 1983; Shipley and Ennis, 1996). One important modulatory pathway to the $\mathrm{OB}$ originates in the nucleus of the horizontal limb of the diagonal band of Broca (HDB), which supplies cholinergic and GABAergic innervation from the basal forebrain system to the MOB (Heimer et al., 1990). Accumulating evidence indicates that olfactory discrimination is at least partially mediated by processes in the OB (Mandairon et al., 2006a, 2008a; Doucette et al., 2007) and that cholinergic centrifugal inputs to the OB modulate perceptual discrimination (De Rosa et al., 2001; Linster and Hasselmo, 2001; Linster and Cleland, 2002; Mandairon et al., 2006b).

To directly assess the relationship between neural and perceptual modulation by cholinergic receptor activation in the $\mathrm{OB}$, we here measure the modulation of mitral cell odorant receptive fields (ORFs) by cholinergic agents infused directly into the olfactory bulb. In a parallel experiment, we measure the effects of the same drug infusions on spontaneous and reward-driven odor discrimination. We find that enhancement of cholinergic modulation in the $\mathrm{OB}$ via local infusions of the acetylcholinesterase inhibitor neostigmine narrows the representation of odorants and increases the number of mitral cells exhibiting differential responses to chemically and perceptually similar odorants. In behavioral experiments, the same enhancement of cholinergic inputs leads to an increase in perceptual discrimination between 
chemically similar odorants. Blockade of both nicotinic and muscarinic receptors in the $\mathrm{OB}$ abolishes the overall effect of neostigmine on neural responses, a result paralleled by a decrease in behavioral discrimination after cholinergic receptor blockade. The present results show, for the first time, a predictive relationship between modulation of neural and perceptual odor discrimination.

\section{Materials and Methods}

\section{Electrophysiological experiments}

Animals. Adult male Sprague Dawley rats (200-250 g) were purchased from Charles River Laboratories. Rats were housed in individual cages with water and food available ad libitum. Animals were maintained in a $12 \mathrm{~h}$ light/dark cycle. All procedures were performed under guidelines established by the Cornell University Institutional Animal Care and Use committee and the National Institutes of Health guidelines.

Experimental preparation. Animals were anesthetized with urethane ( $1.5 \mathrm{~g} / \mathrm{kg}$, i.p.; Sigma) and placed in a stereotaxic apparatus (Narishige). The skull was exposed by scalpel incision, and a hole was drilled over the lateral olfactory tract (LOT) $[+3.7 \mathrm{~mm}$ anteroposterior (AP); $3.4 \mathrm{~mm}$ mediolateral (ML); $6.5 \mathrm{~mm}$ dorsoventral (DV)] and over the olfactory bulbs (5.2 mm AP; $1.8 \mathrm{~mm} \mathrm{ML}$ ). Respiratory activity was monitored throughout the experiments using a piezoelectric monitor strapped around the animal's chest. Signals from the respiratory output were sent to the computer enabling synchronization of odor delivery with inhalation.

Drugs. Three drugs were used in this study: the acetylcholinesterase inhibitor neostigmine $(20 \mathrm{~mm})$, the nicotinic receptor antagonist methyllycaconitine citrate hydrate (MLA) (20 mM), and the muscarinic receptor antagonist scopolamine $(40 \mathrm{~mm})$. All drugs were dissolved in $0.9 \%$ saline, and $6 \mu \mathrm{l}$ were delivered per MOB. The dosage used was based on previously published studies in which the cholinergic drugs were directly infused into the olfactory bulb (Ravel et al., 1994; Mandairon et al., 2006b). A higher dosage of the acetylcholinesterase inhibitor was found to be necessary to obtain significant effects in the anesthetized preparation compared with behavioral experiments, presumably because of the absent or low activation of the cholinergic system in this state. The infusion volume of $6 \mu \mathrm{l}$ had been determined previously to be sufficient to distribute into the whole MOB (Ravel et al., 1994; Mandairon et al., 2006a,b, 2008a) without spilling beyond the main and accessory olfactory bulbs. Drugs were administered $\sim 20$ min before the recording session because this had been shown to be sufficient time for drug action (Mandairon et al., 2006b). Drugs were delivered using a stereotaxic syringe pump (Stoelting) attached to a $50 \mu$ l Hamilton syringe at an infusion rate of $0.50 \mu \mathrm{l} / \mathrm{min}$. At the beginning of the experiment, the syringe was lowered into the $\mathrm{OB}$ targeting the center of the $\mathrm{OB}(+8.0 \mathrm{~mm} \mathrm{AP}$; $\pm 1.5 \mathrm{~mm} \mathrm{ML} ;-5.5 \mathrm{~mm}$ DV from bregma). Previous experiments have shown that a drug volume of $6 \mu \mathrm{l}$ infused at $0.5 \mu \mathrm{l} / \mathrm{min}$ at this position is sufficient to diffuse throughout the $\mathrm{OB}$ without spilling beyond the main and accessory OB (Mandairon et al., 2006a,b).

Odor stimulation. Odorants used were ethyl acetate (E2), ethyl propionate (E3), ethyl butyrate (E4), ethyl valerate (E5), and the carrier control mineral oil (MO). These odorants are part of a family of odorants shown to produce perceptual similarity gradients: as the difference in carbons between two odorants increases, their perceptual similarity also decreases (Linster and Hasselmo, 1999; Linster et al., 2001a; Cleland et al., 2002; Cleland and Narla, 2003). Odorants were delivered by passing a stream of humidified, charcoal-filtered air and mixed with a stream of specific pure humidified odorants (1:50 dilution). The odor protocol consisted of a $2 \mathrm{~s}$ odor delivery separated by a $60 \mathrm{~s}$ interstimulus interval. Each odor was presented five times, and odors were presented in pseudorandom order.

Electrophysiological recordings. Bipolar stimulating electrodes (100 $\mu \mathrm{m}$ stainless steel, Formvar insulated) were placed in the LOT to evoke antidromic action potentials. Unit recordings and extraction of action potentials followed standard methods (Wilson, 2000; Fletcher and Wilson, 2003). Stimulation currents ( $100 \mathrm{~ms}$ duration, 200-900 $\mu \mathrm{A}$ ) were delivered by a constant-current stimulus isolation unit (model PSIU6) con- trolled by a Grass S88 stimulator (Grass-Telefactor). Neuronal responses were recorded using a monopolar tungsten electrode (5-10 M 2 ; A-M Systems). Electrodes were lowered into the mitral cell layer using a fine control micromanipulator (David Kopf Instruments). Optimal placement of the recording electrode into the mitral cell layer of the MOB was achieved by monitoring the size and shape of field potentials (amplified $1000 \times$; low-pass filter, $0.1 \mathrm{~Hz}$; high-pass filter, $475 \mathrm{~Hz}$; sampled at 20 $\mathrm{kHz}$ ) after LOT stimulation (for details, see Haberly, 1973). Single units (amplified 5000×; low-pass filter, $600 \mathrm{~Hz}$; high-pass filter, $6 \mathrm{kHz}$; sampled at $20 \mathrm{kHz}$ ) were recorded in the dorsomedial region of the $\mathrm{OB}$ known to be responsive to ethyl esters of differing hydrocarbon chains (Wilson, 2000; Fletcher and Wilson, 2003). Single units were recorded using the Spike 2 software (Cambridge Electronics Design). Single units were initially isolated online and more rigorously extracted by offline template matching.

Spike sorting. Single units were extracted offline using Spike 2 software. First, templates of spikes were selected from the raw data and then extracted from the whole dataset. Extracted spikes were separated through principal component analysis (PCA; Spike 2 software) whereby features from the data are extracted and clustered to groups of similar waveforms. PCA allows the removal of "noise" and the separation of two or more separate spikes that may have been detected by the electrode. After spike sorting, the number of spikes were automatically counted $4 \mathrm{~s}$ before and $4 \mathrm{~s}$ after each odor onset.

Histological verification of electrode placements. At the end of the experiment, positive current was passed through the electrodes $(5 \mathrm{~s}$ via a $9 \mathrm{~V}$ battery) to produce a small lesion. Transcardial perfusion was then performed with saline and 10\% Formalin. Brains were sectioned at $40 \mu \mathrm{m}$ and subsequently stained with neutral red for electrode localization.

Data analysis. We first determined whether a given cell exhibited significant changes in firing rate after exposure to at least one odor by comparing the number of spikes $4 \mathrm{~s}$ before odor delivery with that $4 \mathrm{~s}$ after odor onset (paired $t$ test, $p=0.05$ ) (Wilson, 2000). Cells that responded significantly to at least one of the odorants were used in all subsequent analyses. Among these cells, we then determined whether the response of any given cell was significantly dependent on odor identity under each drug condition (ANOVA with the difference between preodor and post-odor response as main measure and odor as main effect) and whether the cell differentiated between pairs of odorants [post hoc comparisons, Fisher's least significant difference (LSD) test with $p=$ 0.05]. For comparisons between the different drug conditions, an ANOVA on the proportion of cells that differentiated between two odorants was performed, followed by post hoc pairwise comparisons (Fisher's LSD test) to determine which drug group was significantly different from the others.

\section{Behavioral experiments}

Subjects. Eight male Sprague Dawley rats (250-300 g) were obtained from Charles River Laboratories. Rats were kept alone in standard laboratory cages on a $12 \mathrm{~h} \mathrm{light/dark} \mathrm{cycle} \mathrm{at} \mathrm{a} \mathrm{constant} \mathrm{temperature.} \mathrm{During}$ the testing of spontaneous discriminations (experiment 1), rats were allowed access to food and water ad libitum. During the reinforced olfactory discrimination task (experiments 2), rats were maintained on a food-deprivation schedule designed to keep them at $\sim 85-95 \%$ of their body weight over the behavioral testing period. Water was continuously available. Behavioral experiments were conducted in the afternoon (1400-1800). All procedures were performed under the auspices of a protocol approved by the Cornell University Institutional Animal Care and Use Committee.

Behavioral shaping. Rats were shaped to perform the digging task used for the food-reinforced tasks before cannulation. Rats were first exposed to the two-chambered experimental box used for the procedure over several days until they became accustomed to it, and then they were taught to retrieve a reward (General Mills Frosted Cheerios, preexposed to heat and air to reduce odor) by digging in dishes of corncob bedding (Bed-O-Cobs; The Andersons).

Cannulation. Rats were anesthetized with an intramuscular mixture injection of $50 \mathrm{mg} / \mathrm{kg}$ ketamine and $7.5 \mathrm{mg} / \mathrm{kg}$ xylazine (in a volume of 1 $\mathrm{ml} / \mathrm{kg}$ ) and secured in a stereotaxic instrument (Narishige). Cannulas (22 
Table 1. 0dorsets and dilutions used for behavioral experiments

\begin{tabular}{|c|c|c|c|c|}
\hline & $\mathrm{O}_{\text {hab/cond }}$ & $O_{\text {test1 }}$ & $0_{\text {test2 }}$ & $0_{\text {test3 }}$ \\
\hline Acids & Acetic acid (0.007) & Propionic acid (0.0332) & Butyric acid (0.128) & Hexanoic acid (0.64330) \\
\hline Acetate ester & Ethyl acetate (0.0016) & Propyl acetate $(0.0062)$ & Butyl acetate $(0.0128)$ & Hexyl acetate (0.054) \\
\hline Aldehydes & Butanal $(0.0018)$ & Pentanal (0.066) & Hexanal (0.0222) & Heptanal (0.071) \\
\hline Acetates & Butyl acetate $(0.0218)$ & Butyl propionate (0.0694) & Butyl butyrate (0.1652) & Butyl hexylate (0.7650) \\
\hline
\end{tabular}

Values in parentheses are percentage dilution.

Table 2. Summary of spontaneous discrimination results

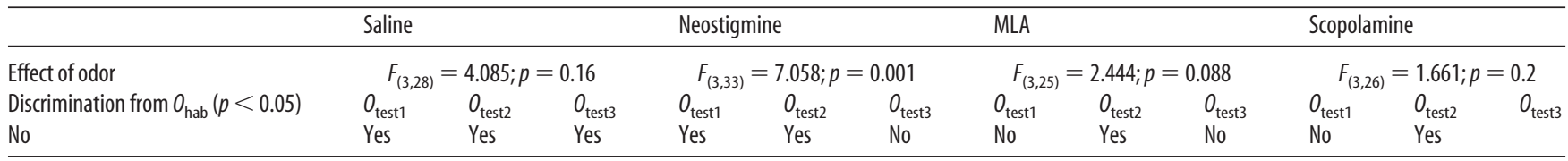

For saline, $F_{(3,28)}=4.085, p=0.16$; for neostigmine, $F_{(3,33)}=7.058, p=0.001$; for $M L A, F_{(3,25)}=2.444, p=0.088$; for scopolamine, $F_{(3,26)}=1.661, p=0.2$.

gauge; Plastics One) were inserted bilaterally into the OBs according to standard procedures (Mandairon et al., 2006a,b, 2008b). Cannulas were implanted at the following coordinates with respect to bregma: $+8.0 \mathrm{~mm}$ AP; $\pm 1.5 \mathrm{~mm} \mathrm{ML;}-4.5 \mathrm{~mm}$ DV. The tips of the guide cannulas were positioned $1 \mathrm{~mm}$ dorsal to the target infusion site; consequently, infusion cannulas extended $1 \mathrm{~mm}$ from the end of the guide cannulas. Five screws were drilled into the skull, and dental cement was used to secure the guide cannulas and cover the incision area. Dummy infusion cannulas were then placed into the guide cannulas to prevent blockage or infection. After surgery, rats were allowed to recover for $10 \mathrm{~d}$. For drug administration, two infusion cannulas were fitted into the guide cannulas so that their tips protruded $1.0 \mathrm{~mm}$ beyond the ends of the guide cannulas into the center of each MOB. Two $10 \mu \mathrm{l}$ Hamilton syringes containing either drug solutions or vehicle (plain saline) were attached to the cannulas with a polyethylene tube and driven with paired infusion pumps (YA-12 Genie pumps; Kent Scientific).

Drug administration. Three cholinergic agents were used in this study: the nicotinic receptor antagonist MLA $(19.0 \mathrm{~mm}, 6 \mu \mathrm{l}$ delivered per MOB; obtained from Tocris Cookson), the muscarinic receptor antagonist scopolamine ( $38.0 \mathrm{~mm}, 6 \mu$ l delivered per OB; obtained from SigmaAldrich), and the blood-brain barrier-impermanent acetylcholinesterase inhibitor neostigmine $(8.7 \mathrm{~mm}, 6 \mu \mathrm{l}$ delivered per MOB; SigmaAldrich). Infusion volume and drug concentrations were those determined to yield significant effects in a previous study (Mandairon et al., 2006b). Drugs were delivered bilaterally into awake rats at a rate of 2 $\mu \mathrm{l} / \mathrm{min}$ for $3 \mathrm{~min}$ ( $6 \mu \mathrm{l}$ total volume delivered per side). The infusion cannulas remained in place for 1 additional minute after the infusion ended to minimize backflow. Behavioral testing was performed $20 \mathrm{~min}$ after drug administration was completed.

Odor sets. To allow for repeated testing under all four drug conditions without repeatedly presenting the same odorants, four odor sets were used for the behavioral experiments (Table 1). Each odor set consisted of a homologous series of aliphatic molecules with unbranched carbon chains presenting the same perceptual characteristics as those used in the electrophysiological experiments: odorants are perceived as increasingly dissimilar from each other as the difference in carbon chain length increases (Linster and Hasselmo, 1999; Cleland et al., 2002; Cleland and Narla, 2003). The three test odorants in each odor set were used for all drug conditions to allow for higher resolution of drug effects compared with our previous study (Mandairon et al., 2006b). All odorants were diluted in mineral oil so as to theoretically emit a steady-state vapor phase partial pressure of 1.0 $\mathrm{Pa}$ (Cleland et al., 2002). For each experiment, rats were exposed to each odor set only once. Experiments were separated by 2 weeks from each other to prevent interference between experiments.

Experiment 1: habituation learning and spontaneous discrimination

An olfactory cross-habituation task assesses the degree to which rats spontaneously discriminate odorants by habituating them to one odorant $\left(O_{\text {hab }}\right)$ and then measuring the magnitude of their novelty response to a second odorant $\left(O_{\text {test }}\right)$. Presentation of a novel odorant will elicit substantial investigation of the new stimulus; however, if the second odorant is perceived as similar to the habituated odorant (i.e., it is poorly discriminated), the rat will spend less time investigating the second odorant when it is presented. Because no reward is associated with either odorant in this task, and each test odor is presented only once, this task is used to measure baseline perceptual similarities between odorants, unaltered by a history of reinforcement. All habituation experiments took place in the home cages of the test animals under red light. All odors were diluted in mineral oil according to Table 2 and prepared before each experiment; odor sets were double blinded so that the experimenter was unaware of the identity of each odor. Odors were presented by placing 60 $\mu l$ of the odor stimulus onto a filter paper disc (Whatman \#1) contained within a weighing dish that was placed on top of the wire cage lid. This procedure enabled the observer to change the odor stimulus without unduly disturbing the animal. Each test session was preceded by one $50 \mathrm{~s}$ presentation of plain mineral oil. Test sessions comprised four $50 \mathrm{~s}$ presentations of the habituation odor at 5 min intervals, followed by three $50 \mathrm{~s}$ presentations of test odorants in randomized order. The amount of time that the rat spent actively investigating each presented odorant was measured. Active investigation was defined as directed sniffing within 1 $\mathrm{cm}$ of the odor source. Individual rats were repeatedly tested under different drug conditions but encountered different odor sets under each condition to prevent rats from becoming familiar with the odorants. The order of presentation of odor sets and the pairing of odor sets with drug conditions were counterbalanced.

Data analysis. Data analyses were performed using SPSS statistical software with the time spent investigating odor stimuli during presentation trials as the dependent variable. Because habituation is necessary to assess discrimination ANOVA was performed across the four habituation trials, followed by Fisher's post hoc pairwise comparisons to determine whether rats in each drug treatment group habituated to the habituation odor (significant decrease in investigation time between the first and fourth habituation trials). Separate ANOVAs followed by Fisher's post hoc pairwise comparisons were subsequently performed to determine whether rats in each drug treatment group (1) exhibited reduced spontaneous discrimination between habituated and test odors (one, two, or three carbon difference from habituated odor) compared with vehicle-injected animals, and (2) discriminated significantly between the habituation and test odors, as indicated by a significant increase in investigation time during the test trial when compared with the fourth habituation trial. The criterion for significance was set to $\alpha=0.05$.

\section{Experiment 2: odor reward conditioning, strength, and specificity} of association

In this experiment, we tested how discrimination between a conditioned and novel test odorant is modulated by cholinergic inputs using the same odor sets as in experiment 1 .

Rats were conditioned to the conditioned odor $\left(O_{\text {cond }}\right)$ by retrieving a reward buried in a scented dish during eight successive trials in which it had a choice between a scented dish containing the reward and an unscented dish containing no reward. The strength and precision of the odor-reward association was then tested in four unrewarded test trials during which time rats spent looking for the reward in the conditioned 
B

A

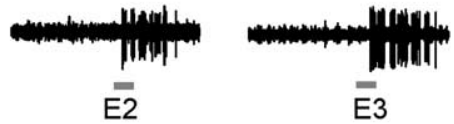

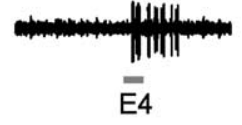

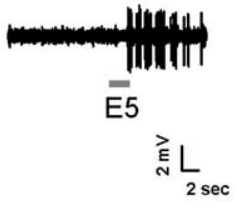

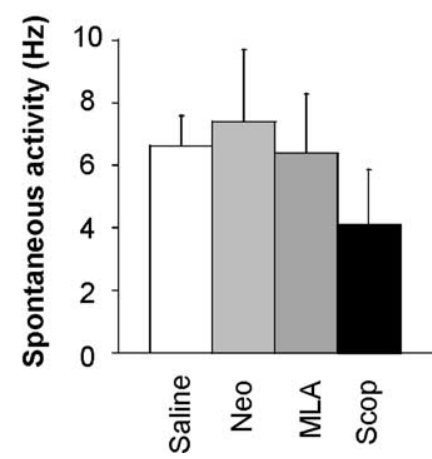

Figure 1. A, Examples of single-trial responses of a select mitral cell to each of the four odorants under saline conditions. $\boldsymbol{B}$, Comparison of spontaneous activity between saline and drug conditions. Neo, Neostigmine; Scop, scopolamine.

odor and the three test odors was recorded. This task, described in detail previously, assesses the degree to which rats associate a reward with an odorant after several training trials as well as to what degree this association is odor specific (Linster and Hasselmo, 1999; Cleland et al., 2002). Each odorant was tested a single time under a different drug condition. The experimenter was blind to the identity of the test odors during performance of these experiments.

Data analysis. The primary data, as depicted on figure ordinates, consisted of digging times during test trials. After ANOVA testing for differences in digging times among rats, using test odorant as a within-subject factor (differences of zero, one, two, or three carbons from $O_{\text {cond }}$ ) and drug condition as between subjects factor, post hoc tests (Fisher's LSD test) were performed to determine whether digging times differed as a result of drug treatment for specific test trials and whether the digging time elicited by a test odorant was significantly different from that elicited by $O_{\text {cond. }}$. All tests were two tailed, and $\alpha$ was set to 0.05 . Error bars depict SEs. All statistical analyses were performed using SPSS statistical software.

\section{Results}

\section{Electrophysiological experiments}

In these experiments, we investigated how cholinergic receptor activation modulates mitral cell odor responses and how this modulation is related to pairwise odor discrimination at the level of mitral cell odor representation. We first describe the effects on individual mitral cells. We then discuss how the population response (representation) to the odor series is modulated.

\section{Mitral cell odor responses}

We recorded a total of 110 mitral cells from 38 rats. The majority of mitral cells recorded in our study exhibited significant responses to at least one of the four odors used (71 mitral cells or $65 \%)$. Of the 71 cells that responded significantly to odorants, 45 mitral cells $(66 \%)$ always exhibited excitatory responses, whereas 26 mitral cells (38\%) exhibited a combination of excitatory and inhibitory responses. Of the 71 cells, 26 mitral cells (38\%) responded to only one ester odor, 7 mitral cells (12\%) responded to two ester odors, 16 mitral cells $(24 \%)$ responded to three ester odors, and 23 mitral cells (34\%) responded to all four ester odors. Figure $1 A$ shows a raw recording trace.

\section{Effects of drugs on mitral cell spontaneous activity}

Although the frequency of baseline spontaneous activity can vary between mitral cells, neostigmine, MLA, and scopolamine were not found to modulate spontaneous activity of mitral cells when compared with saline control $\left(F_{(3,44)}=0.322 ; p>0.05\right)$ (Fig. $\left.1 B\right)$.
Effect of cholinergic modulation on mitral cell odor responses Mitral cells were found to exhibit a variety of response changes after modulation of the cholinergic pathway. Neostigmine was typically found to increase the ability of mitral cells to exhibit differential responses to chemically similar odors as seen in the example in Figure $2 A_{i}$. Figure $2 A_{i i}$ shows a mitral cells for which there was no significant difference between odors in either saline or neostigmine conditions.

Overall, neostigmine was found to sharpen mitral cell ORFs: in the presence of neostigmine, $61 \%$ of cells $\left(n_{(\text {Total })}=36\right)$ exhibited significant response differences between odors differing by a single carbon compared with $30 \%$ of cells in saline conditions $\left(n_{\text {(Total })}=71\right)$ [Fig. $2 \mathrm{~B}$; control saline data were all pooled together for data analysis because there was no difference in any of the saline runs (data not shown)]. Neostigmine-dependent sharpening of ORFs was attenuated by the presence of the nicotinic antagonist MLA and to a lesser degree by the muscarinic antagonist scopolamine (Fig. 2 B). Statistical comparisons of the number of cells that exhibited significant response differences between odors differing by a single carbon showed a significant effect of drug (ANOVA; $\left.F_{(5,161)}=2.4 ; p<0.05\right)$. Post hoc analysis showed that, in the presence of neostigmine $(n=36)$ alone, the percentage of mitral cells that exhibited significant response differences between the nearest neighbor odor pairs was significantly greater than that measured in saline $(n=71)$ or neostigmine/MLA $(n=32)$ conditions $(p<0.05)$. Interestingly, there was no significant difference between neostigmine and neostigmine/scopolamine $(n=17)$ conditions $(p>0.05)$. Addition of MLA $(n=7)$ or scopolamine $(n=4)$ alone did not significantly alter the response differences to these odor pairs $(p>0.05)$. The proportion of cells that showed a significant increase in firing in response to the odor chemically most similar to the "best odor" was significantly reduced under neostigmine, whereas the proportion of cells that were significantly inhibited by this odor was significantly increased $(p<0.05)$ (Fig. $2 C$ ). This comparison with the best response clearly shows that, overall, response profiles are sharpened by neostigmine.

Statistical comparisons for the effects of drugs on the ability of the cells to fire differentially in response to odors differing by two carbons or three carbons did not show a significant drug effect (two carbon difference: $F_{(5,161)}=1.6, p>0.05$; three carbon difference: $\left.F_{(5,161)}=0.59, p>0.05\right)$, showing that the experimental manipulation of cholinergic actions performed here modu- 
$A_{i}$

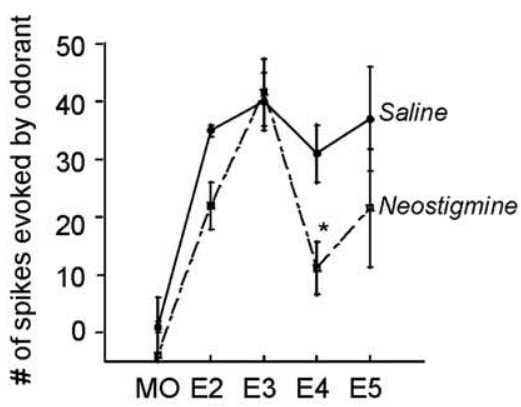

$\mathbf{A}_{\mathrm{ii}}$

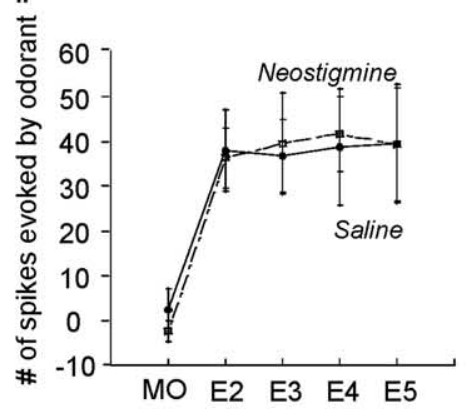

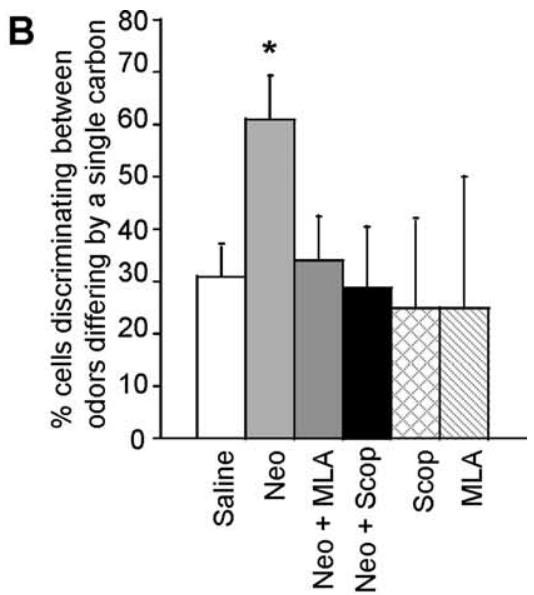

$\mathbf{C}_{\mathbf{i}}$

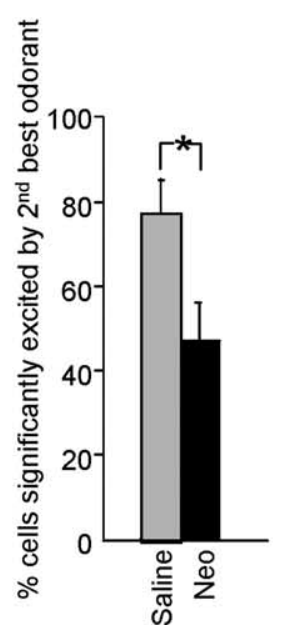

$\mathrm{C}_{\mathrm{ii}}$

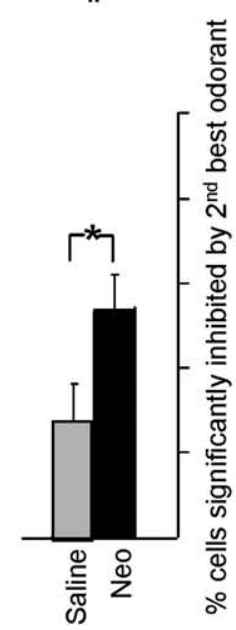

D

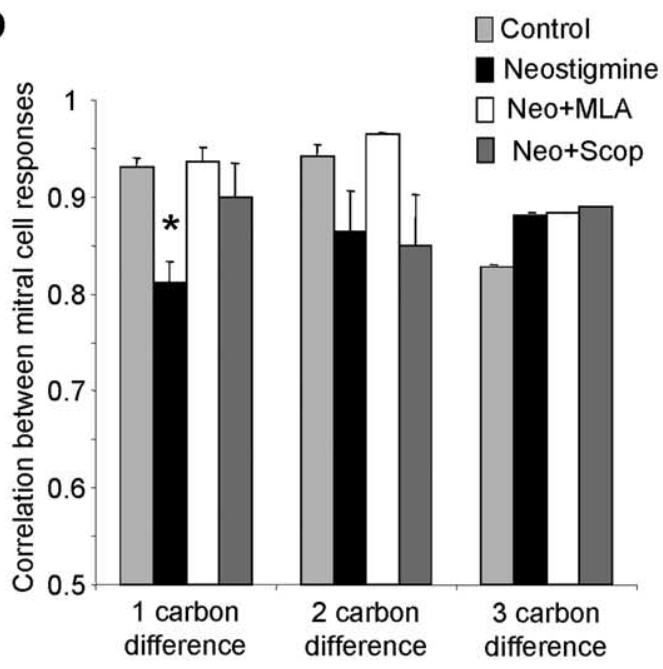

Figure 2. Effect of cholinergic modulation in odor responses. Ai, Aii, Examples of mitral cell odor response modulation by application of neostigmine. The graphs show the number of spikes evoked by odor stimulation (difference in the number of spikes recorded during $4 \mathrm{~s}$ after odor onset and $4 \mathrm{~s}$ before odor onset) in response to the four stimulation odorants (E2-E5). Ai, In this cell, there was no statistical response difference between the four odors $\left(F_{(3,16)}=0.34 ; p>0.05\right)$ in saline. However, in the same cell after infusion of neostigmine, there was sharpening of the 0 RF after exposure to the same four odors $\left(F_{(3,16)}=3.7 ; p<0.05\right)$. Post hoc analysis shows significant differences between E3/E4 $(p<0.01)$ and E3/E5 $(p<0.05)$. $A_{i i}$ shows a mitral cell for which there was no significant difference between odors in either saline or neostigmine conditions (saline, $F_{(3,16)}=0.012, p>0.05$; neostigmine, $\left.F_{(3,16)}=0.049, p>0.05\right)$. B, Number of individual mitral cells exhibiting significantly different responses to odorants differing by one carbon under the control and drug conditions. ${ }^{*} p<0.05$, significant difference between saline and drug conditions. $C$, Proportion of cells responding with significant excitation (Ci) or inhibition (Cii) to the odor chemically most similar to the "best odor" of the cell under saline and neostigmine conditions. ${ }^{*} p<0.05$, significant difference between saline and neostigmine conditions. $\boldsymbol{D}$, Comparison of population responses to pairs of odorants as a function of drug treatment. The graph shows the average correlation between mitral cell responses to odorants differing by one, two, or three carbons. ${ }^{*} p<0.05$, significant reduction in correlation between odor pairs differing by one carbon under neostigmine conditions. Neo, Neostigmine; Scop, scopolamine.

lated the discrimination of chemically very similar (i.e., differing by one carbon) odors only.

To further illustrate the enhanced discrimination between chemically similar odorants by mitral cell spiking responses, we calculated the correlation between mitral cell responses to pairs of odorants differing by one, two, or three carbons. If mitral cells respond similarly to a pair of odorants, the correlation should approach one, and, as their responses differ more, the response correlations take on smaller values. Figure $2 C$ shows the average correlations between responses to pairs of odorants differing by one, two, and three carbons for saline and neostigmine conditions. Note the significant reduction in correlation $(p<0.01, t$ test) under neostigmine, indicating that infusion of neostigmine reduces the similarity in mitral cell responses to these pairs of odorants.

In summary, increasing cholinergic modulation in the $\mathrm{OB}$ by local infusion of neostigmine significantly increased the proportion of mitral cells firing differentially in response to chemically very similar odorants. The observed increase was significant only when odors differing by a single carbon were compared. The neostigmine-induced increase was significantly reduced by the addition of the nicotinic antagonist MLA as well as the muscarinic antagonist scopolamine.

\section{Behavioral experiments}

In these experiments, to understand the effect of mitral cell response modulation on odor perception, we tested the effects of increasing and decreasing cholinergic receptor activation on the discrimination of series of aliphatic odorants differing by increasing number of carbons. Two behavioral tasks were used: a habituation task in which memory formation is non-associative and a reinforced task in which memory formation is in association with a food reward. Both tasks 

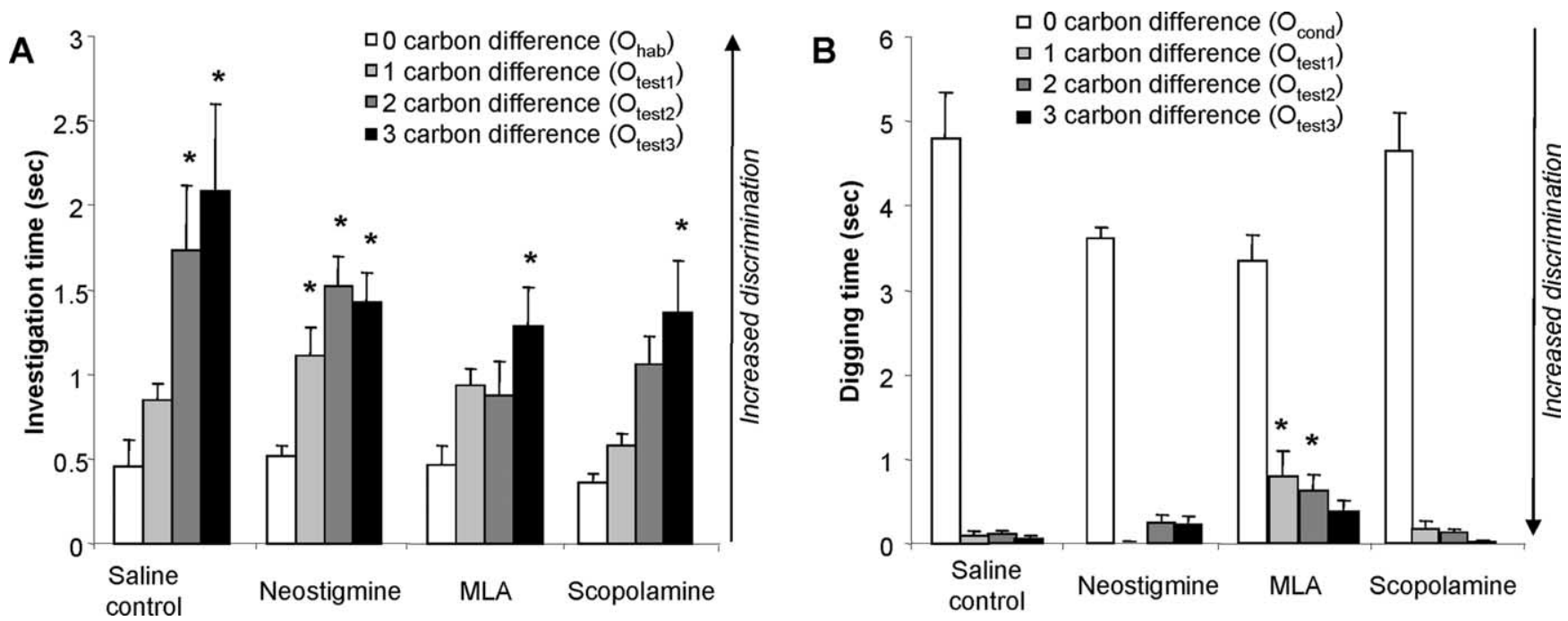

Figure 3. Behavioral experiments. A, Habituation/spontaneous discrimination. Average investigation time during randomized test trials for all drug conditions. ${ }^{*} p<0.05$, significant difference between a response to a test odor and response to the last habituation trial (habituated odor). $\boldsymbol{B}$, Generalization learning, strength, and specificity of association. The graph shows the average digging times in response to the conditioned and test odors for all drug conditions. ${ }^{*} p<0.05$, significant difference compared with saline controls.

use a single conditioning odor and allow to test the specificity of the formed memory by comparing the response to the conditioned odor with that to novel test odorants.

\section{Experiment 1: habituation learning and spontaneous discrimination}

In this experiment, rats are initially habituated to a habituation odorant, after which their investigation response to the habituated and chemically similar test odorants $\left(O_{\text {test } 1-3 \text {, differing }}\right.$ by one, two, or three carbons from $O_{\text {hab }}$ ), presented in randomized order, is recorded (Fig. 3A). This task enables measurement of short-term olfactory memory (i.e., the recognition of the habituation odor over the course of repeated trials) as well as the degree of spontaneous discrimination between chemically similar odorants (i.e., the recognition of a novel test odorant as being different from the habituate odorant).

No significant effect of drug treatment on investigation time during habituation trials was measured $\left(F_{(3,132)}=1.265 ; p>\right.$ $0.2)$; however, a significant effect of trial number $\left(F_{(3,132)}=\right.$ 13.459; $p<0.001)$, without a significant interaction between drug and trial number $\left(F_{(9,132)}=1.408 ; p>0.1\right)$, indicated that all groups of rats decreased their response to repeated odor presentations. Post hoc tests (Fisher's LSD test) showed a significant effect of trial number in all drug groups $(p<0.01$ in all cases), and all rats responded significantly less during the fourth trial compared with the first trial ( $p<0.05$ in all cases).

Comparison of investigation responses during the last presentation of the habituated $\left(O_{\text {hab }}\right)$ and test odors $\left(O_{\text {test1-3 }}\right)$ during the randomized test trials yielded a significant effect of drug treatment $\left(F_{(3,112)}=3.757 ; p<0.02\right)$ as well as a significant effect of odor $\left(F_{(3,112)}=11.057 ; p<0.001\right)$. Pairwise comparisons of investigation responses in each group showed, as predicted from previous experiments, that saline-treated control rats discriminated between odors differing by two or three carbons but not between odors differing by one carbon. Rats in which cholinergic modulation was enhanced (in the presence of neostigmine) were able to discriminate between the habituated odor and all three test odors, showing that enhanced cholinergic inputs facilitate the discrimination of odorants differing by a single carbon. In contrast, rats in which nicotinic receptors were blocked discrimi- nated only the most dissimilar odor from the habituated odor $\left(\mathrm{O}_{\text {test } 3}\right.$, three-carbon difference $)$, as did rats treated with the muscarinic receptor blocker scopolamine. Table 2 summarizes the results by indicating drug conditions under which a significant effect of test odor was observed and which odor pairs were discriminated under each condition. Figure $3 A$ shows the average investigation responses during the last habituation trial and all test trials for all drug conditions.

In summary, compared with saline control, increased activation of cholinergic receptors increased odor discrimination, whereas blockade of muscarinic or nicotinic receptors decreased odor discrimination (Fig. 3A, Table 2).

Experiment 2: odor reward learning, strength, and specificity of association

In this experiment, rats were first conditioned to associate a given odorant $\left(O_{\text {cond }}\right)$ with a food reward (Fig. $\left.3 B\right)$. The strength and specificity of the formed association was then tested during nonrewarded test trials with the conditioned odor $\left(O_{\text {cond }}\right)$ as well as with several chemically similar test odorants $\left(O_{\text {test1-3 }}\right)$. ANOVA using digging time during non-rewarded test trials as variable showed no effect of treatment group $\left(F_{(3,267)}=0.845 ; p>0.5\right)$ but an effect of test odor $\left(F_{(3,267)}=150.816 ; p<0.001\right)$ as well as significant interaction between these two effects $\left(F_{(9,267)}=2.335\right.$; $p<0.02)$, thus indicating that differences in relative digging times may be attributed to cholinergic modulation.

Additional statistical testing showed that a significant effect of treatment group on digging times was not found for the conditioned odor $\left.\left(F_{(3,66}\right)=1.630 ; p>0.1\right)$, indicating that the response to the conditioned odor (i.e., strength of odor-reward association) was not affected by the drug treatment. A significant effect of treatment group was found among the responses to test odors $O_{\text {test } 1}$ (one-carbon difference; $F_{(3,64)}=3.117 ; p<0.05$ ) and $O_{\text {test2 }}$ (two-carbon difference; $F_{(3,71)}=3.298 ; p<0.05$ ) but not $O_{\text {test3 }}$ (three-carbon difference; $F_{(3,66)}=2.230 ; p>0.05$ ). Specifically, rats treated with the nicotinic antagonist MLA responded significantly more to test odors differing by one or two carbons from the conditioned odor, showing that these rats discriminated less between these odor pairs (Fig. 3B).

In summary, these results show that blockade of bulbar nico- 
A

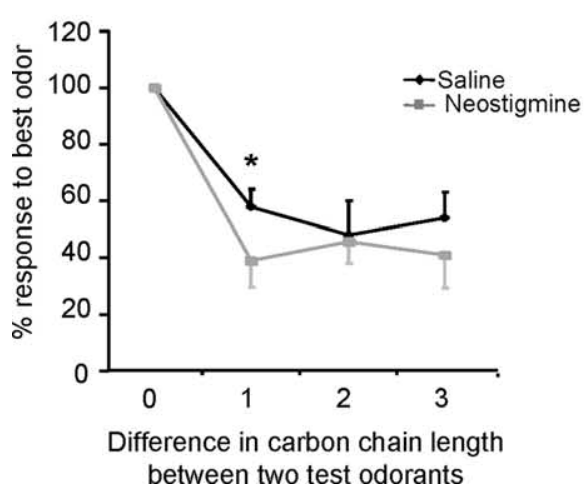

B

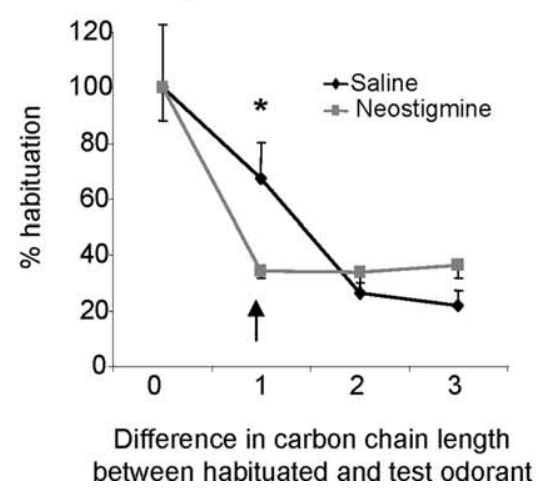

C Odor reward learning

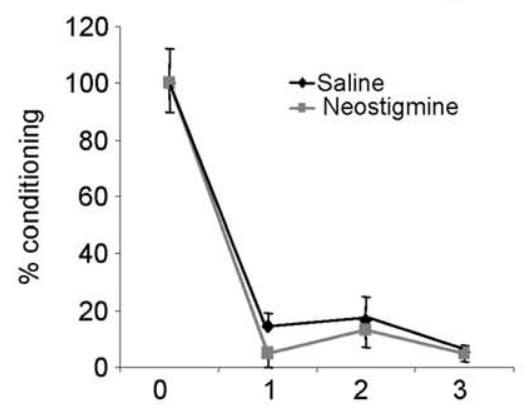

Difference in carbon chain length Between conditioned and test odorant

Figure 4. Comparison between electrophysiological and behavioral results. $\boldsymbol{A}$, Average normalized olfactory response functions of recorded mitral cells. All responses are shown as the percentage of the best odor response and arranged as a function of carbon chain length difference to the best response odor. A significant difference between saline and neostigmine response functions increases the discrimination between odor pairs differing by a single carbon (indicated by ${ }^{*} p<0.05$ ). $\boldsymbol{B}$, Spontaneous discrimination. The graph shows the percentage of habituation to an odorant with respect to the habituated odorant (difference in carbon chain length 0 , at 100\%). A decrease in habituation signifies an increase in discrimination in this experiment. Note the difference in discrimination between odorants differing by a single carbon between saline and neostigmine conditions (indicated by ${ }^{*} p<0.05$ ). $C$, Discrimination after associative conditioning. The graph shows the percentage of conditioning (100\% being the response to the conditioned odor) as a function of the chain length difference between the conditioned and the test odor. Note the increase in discrimination between odors differing by a single carbon attributable to neostigmine (indicated by ${ }^{*} p<0.05$ ).

tinic receptors decreased discrimination between the conditioned odor and chemically similar odorants differing by one or two carbons (Fig. 3B).

\section{Comparison between electrophysiological and behavioral experiments}

To compare electrophysiological and behavioral results, we plotted the population ORFs, i.e., the average response of all recorded mitral cells, normalized by the best response of each mitral cell, as a function of the difference in carbon chain length between the best response odor and the other odor stimuli. Hence, the graph allows us to visualize how the response of mitral cells changes as a function of carbon chain length difference between the odorant that evokes the best or strongest response in a given cell and subsequent odorants differing by one, two, and three carbons (Fig. 4A). The graph depicts the population ORF under saline control and neostigmine conditions, showing that neostigmine significantly enhances the difference between odorants differing by a single carbon $(p<0.05$, denoted by $\left.{ }^{*}\right)$. Spontaneous discrimination, graphed as the percentage habituation of test odorants (with respect to the habituation odorant), is shown as a function of difference in carbon chain length between the habituated and test odorant (Fig. $4 B$ ). The graph shows that spontaneous discrimination is enhanced between odorants differing by a single carbon under neostigmine conditions $(p<0.05$, denoted by $\left.{ }^{\star}\right)$. Similarly, discrimination between odorants differing by a single carbon after odor reward conditioning is enhanced under neostigmine conditions, albeit to a lesser degree (Fig. 4C). The graph shows the percentage of conditioned response to a test odorant with respect to the conditioned odor as a function of the difference between conditioned and test odor under saline and neostigmine conditions; the difference observed between saline and neostigmine conditions is denoted with an asterisk.

These graphs clearly illustrate the predictive relationship between cholinergic modulation of neural and behavioral responses.

\section{Discussion}

The present experiments show a correspondence between the modulation of mitral cell odor responses and behavioral odor discrimination by activation of cholinergic receptors in the olfactory bulb.
Activation of cholinergic receptors in the olfactory bulb significantly increased the number of mitral cells responding differentially to pairs of aliphatic odorants differing by a single carbon in their chain as well as behavioral discrimination between these odorant pairs. Cholinergic pathways have traditionally been associated with attentional mechanisms: in the present framework, increased cholinergic activity enhances perceptual discrimination by changing neural stimulus representations, a phenomenon that can be interpreted as increased attention.

The organization of the olfactory system is such that spatially distributed olfactory sensory neurons, expressing common olfactory receptors, converge unto one or a few common glomeruli. Localization of mitral cells that respond to families of odorants are shown to be distributed in particular regions of the OB (Johnson et al., 1999; Mori et al., 1999). For example, mitral cells in particular regions of anesthetized rabbit $\mathrm{OB}$ were found to preferentially respond, by increase or decrease in spike frequency, to unbranched aliphatic aldehydes of specific number of carbon chain length (Imamura et al., 1992; Yokoi et al., 1995). In general, odorants with similar carbon chain lengths will activate overlapping populations of glomeruli and mitral cells more so than odorants with dissimilar carbon chain lengths (Imamura et al., 1992; Meister and Bonhoeffer, 2001; Johnson et al., 2004; Farahbod et al., 2006). Mitral cells responding to these odorants are often sparsely and widely distributed across the corresponding olfactory bulb area (Davison and Katz, 2007; Fantana et al., 2008). Mitral cells interact with each other via local interneurons, many of which are the targets of central and neuromodulatory inputs (for review, see Shipley and Ennis, 1996), enabling the modulation of odor representations by these central and neuromodulatory inputs.

The $\mathrm{OB}$ receives strong cholinergic inputs from the basal forebrain via the nucleus of the HDB that innervates primarily glomerular and granule cell layers of the bulb (Heimer et al., 1990; Kasa et al., 1995). Activation of cholinergic receptors in the OB acts on both nicotinic and muscarinic receptors (Castillo et al., 1999; Ghatpande et al., 2006; Pressler et al., 2007); these are well segregated with a low degree of overlap, whereby nicotinic receptors are typically located within the glomerular and mitral cell 
layer, whereas muscarinic receptors predominate the plexiform and granule cell layers. Cholinergic neuromodulation in the $\mathrm{MOB}$, including both nicotinic and muscarinic components, has been hypothesized previously to regulate the discrimination of similar odorants (for review, see Linster and Cleland, 2002). For example, intraperitoneal injections of physostigmine, an acetycholinesterase inhibitor, were found to enhance odor discrimination between extremely similar odorant stimuli in a rewarded forced-choice task (Doty et al., 1999), lesions of cholinergic inputs to the $\mathrm{OB}$ and cortex reduced the differentiation of chemically similar odorants (Linster et al., 2001a; Linster and Cleland, 2002), and direct infusion of cholinergic agents into the MOB modulated spontaneous discrimination of chemically similar odorants (Mandairon et al., 2006b). Our electrophysiological findings show that odor discrimination is enhanced at the level of mitral cell spiking responses when cholinergic receptors are activated during odor stimulation (Fig. 2). Our behavioral observation shows that enhancement of bulbar cholinergic modulation increases olfactory discrimination (Fig. 3). Blockade of both nicotinic and muscarinic receptors abolished the enhanced discrimination observed in mitral cells, suggesting that both receptor types participate in the modulation of mitral cell responses. Behavioral experiments support these results in that they show that blockade of both receptor types decreases spontaneous discrimination, more particularly the discrimination between aliphatic odorants differing by one or two carbons. In contrast, in a reward-conditioned task, blockade of muscarinic receptors did not affect odor discrimination, whereas blockade of nicotinic receptors did. Interestingly, a previous experiment from our group, using a more limited odor set and a different conditioned task, showed that addition of a muscarinic antagonist to a low dosage of a nicotinic antagonist, not effective by itself, decreased spontaneous discrimination between chemically similar odorants (Mandairon et al., 2006b). Given that the electrophysiologically measured effects on odor discrimination are weaker for muscarinic receptor blockade (no significant difference between neostigmine and scopolamine induced effects on the number of cells discriminating between chemically similar odorants), it may be that the role of muscarinic receptors is secondary to those of nicotinic receptors and can only be seen in the absence of rewardmediated motivation or when nicotinic action has been disturbed. Overall, the effects of cholinergic blockade were much larger when spontaneous, unmotivated discrimination was tested, supporting the idea that the activation of cholinergic receptors in the olfactory bulb shapes odor representations, in a manner that affects perception but not to the extent of preventing the learning of an odor-reward association (Figs. 3, 4). However, potential benefits of increased cholinergic action in the MOB may not appear in the reward-motivated behavioral task because control rats do not significantly confuse the conditioned and test odorants. These data are supported by previous experiments showing that pairs of odorants not spontaneously discriminated by rats can be rendered discriminable by reward-driven learning as well as by daily exposure to odorants (Linster et al., 2002; Mandairon et al., 2006a). Reward-driven odor learning may be accompanied by changes in odor representations at multiple levels of the olfactory system and activate plasticity mechanisms other than cholinergic modulation. Global interruption of cholinergic modulation, by either systemic injections or specific lesions of cholinergic neurons, have been shown previously to affect reward-driven learning (Doty et al., 1999; De Rosa et al., 2001; Linster et al., 2001a), suggesting that cholinergic inputs to the olfactory bulb proper, modulated in the present experiments, may underlie different functionalities than those acting on other olfactory centers. More specifically, cholinergic modulation in the $\mathrm{OB}$ would specifically modulate odor representations, whereas cholinergic modulation in olfactory cortical areas have been shown to modulate synaptic plasticity and odor reward learning (for review, see Linster and Hasselmo, 2001).

Brain-slice experiments have shown that activation of nicotinic receptors both directly depolarizes mitral cells while simultaneously increasing their inhibition by periglomerular cells (Castillo et al., 1999). A recent computational model showed that these two phenomena can act together to increase the sparseness of mitral cell odor responses and to increase the degree of discrimination between chemically similar odorants at this level of mitral cell spiking activity (Mandairon et al., 2006b). Periglomerular cells narrow the response curves of individual mitral cells locally without the need for local and later inhibition between glomeruli (Cleland and Sethupathy, 2006); hence, the cholinergic modulation of response profiles in this model is compatible with new data showing sparse and wide distribution of mitral cells responding to chemically similar odorants (Davison and Katz, 2007; Fantana and Meister, 2008). Activation of muscarinic receptors has been reported to inhibit the firing of granule cells while at the same time increasing the degree of activity-dependent GABA transmitter release onto mitral cell secondary dendrites (Castillo et al., 1999). In addition, recent papers showed that muscarinic receptor activation is highly dosage dependent and regulates the response reliability and noise of granule and mitral cells (Pressler et al., 2007) and that muscarinic ACh receptor activation modulates a subset of periglomerular cells (Pignatelli and Beluzzi, 2008) in the glomerular layer. The authors found that muscarinic ACh activation increased membrane hyperpolarization, via potassium channel activation, in dopaminergic periglomerular cells, leading to a decrease in spike activity in modulated cells. Together, the effects of muscarinic receptor activation could result in a more specific effect of inhibition mediated by strongly activated granule cells in regions of responsive mitral cells, acting to regularize and synchronize mitral cell firing in response to odorants, as suggested by several computational models of olfactory bulb function (Lagier et al., 2004; Bathellier et al., 2006; Galán et al., 2006; David et al., 2008). As a consequence, the synergistic result from nicotinic and muscarinic receptor activation is one of limiting mitral cell responsiveness to odorants by increasing inhibitory action at the glomerular tuft, narrowing the olfactory receptive field, while simultaneously delivering specific inhibition at the level of secondary dendrites, stabilizing this reduction of receptive fields as well as increasing response reliability and modulating synchrony (Linster and Gervais, 1996; Linster and Hasselmo, 1997; Linster et al., 1999).

In summary, cholinergic modulation onto $\mathrm{MOB}$ circuitry appears to play a functional role in odor representations. We here show, using coordinated behavioral and electrophysiological experiments, that increasing ACh activity in the OB increases perceptual discrimination in rats and sharpens mitral cell ORFs, respectively.

\section{References}

Bathellier B, Lagier S, Faure P, Lledo PM (2006) Circuit properties generating gamma oscillations in a network model of the olfactory bulb. J Neurophysiol 95:2678-2691.

Castillo PE, Carleton A, Vincent JD, Lledo PM (1999) Multiple and opposing roles of cholinergic transmission in the main olfactory bulb. J Neurosci 19:9180-9191.

Cleland TA, Linster C (2005) Computation in the olfactory system. Chem Senses 30:801-813.

Cleland TA, Narla VA (2003) Intensity modulation of olfactory acuity. Behav Neurosci 117:1434-1440.

Cleland TA, Sethupathy P (2006) Non-topographical contrast enhancement in the olfactory bulb. BMC Neurosci 7:7. 
Cleland TA, Morse A, Yue EL, Linster C (2002) Behavioral models of odor similarity. Behav Neurosci 116:222-231.

Cleland TA, Johnson BA, Leon M, Linster C (2007) Relational representation in the olfactory system. Proc Natl Acad Sci U S A 104:1953-1958.

David F, Linster C, Cleland TA (2008) Lateral dendritic shunt inhibition can regularize mitral cell spike patterning. J Comput Neurosci 25:25-38.

Davison IG, Katz LC (2007) Sparse and selective odor coding by mitral/ tufted cells in the main olfactory bulb. J Neurosci 27:2091-2101.

De Rosa E, Hasselmo ME, Baxter MG (2001) Contribution of the cholinergic basal forebrain to proactive interference from stored odor memories during associative learning in rats. Behav Neurosci 115:314-327.

Doty RL, Bagla R, Kim N (1999) Physostigmine enhances performance on an odor mixture discrimination test. Physiol Behav 65:801-804.

Doucette W, Milder J, Restrepo D (2007) Adrenergic modulation of olfactory bulb circuitry affects odor discrimination. Learn Mem 14:539-547.

Fantana AL, Soucy ER, Meister M (2008) Rat olfactory bulb mitral cells receive sparse glomerular inputs. Neuron 59:802-814.

Farahbod H, Johnson BA, Minami SS, Leon M (2006) Chemotopic representations of aromatic odorants in the rat olfactory bulb. J Comp Neurol 497:350-366.

Fletcher ML, Wilson DA (2003) Olfactory bulb mitral-tufted cell plasticity: odorant-specific tuning reflects previous odorant exposure. J Neurosci 23:6946-6955

Galán RF, Fourcaud-Trocmé N, Ermentrout GB, Urban NN (2006) Correlation-induced synchronization of oscillations in olfactory bulb neurons. J Neurosci 26:3646-3655.

Ghatpande AS, Sivaraaman K, Vijayaraghavan S (2006) Store calcium mediates cholinergic effects on mIPSCs in the rat main olfactory bulb. J Neurophysiol 95:1345-1355.

Haberly LB (1973) Summed potentials in opossum prepyriform cortex. J Neurophysiol 36:775-788.

Halász N, Shepherd GM (1983) Neurochemistry of the vertebrate olfactory bulb. Neuroscience 10:579-619.

Hasselmo ME, Giocomo LM (2006) Cholinergic modulation of cortical function. J Mol Neurosci 30:133-135.

Hasselmo ME, Anderson BP, Bower JM (1992) Cholinergic modulation of cortical associative memory function. J Neurophysiol 67:1230-1246.

Heimer L, Zahm DS, Schmued LC (1990) The basal forebrain projection to the region of the nuclei gemini in the rat; a combined light and electron microscopic study employing horseradish peroxidase, fluorescent tracers and Phaseolus vulgaris-leucoagglutinin. Neuroscience 34:707-731.

Imamura K, Mataga N, Mori K (1992) Coding of odor molecules by mitral/ tufted cells in rabbit olfactory bulb. I. Aliphatic compounds. J Neurophysiol 68:1986-2002.

Johnson BA, Woo CC, Hingco EE, Pham KL, Leon M (1999) Multidimensional chemotopic responses to n-aliphatic acid odorants in the rat olfactory bulb. J Comp Neurol 409:529-548.

Johnson BA, Farahbod H, Xu Z, Saber S, Leon M (2004) Local and global chemotopic organization: general features of the glomerular representations of aliphatic odorants differing in carbon number. J Comp Neurol 480:234-249.

Kasa P, Hlavati I, Dobo E, Wolff A, Joo F, Wolff JR (1995) Synaptic and non-synaptic cholinergic innervation of the various types of neurons in the main olfactory bulb of adult rat: immunocytochemistry of choline acetyltransferase. Neuroscience 67:667-677.

Lagier S, Carleton A, Lledo PM (2004) Interplay between local GABAergic interneurons and relay neurons generates gamma oscillations in the rat olfactory bulb. J Neurosci 24:4382-4392.

Linster C, Cleland TA (2002) Cholinergic modulation of sensory representations in the olfactory bulb. Neural Netw 15:709-717.

Linster C, Gervais R (1996) Investigation of the role of interneurons and their modulation by centrifugal fibers in a neural model of the olfactory bulb. J Comput Neurosci 3:225-246.

Linster C, Hasselmo M (1997) Modulation of inhibition in a model of olfactory bulb reduces overlap in the neural representation of olfactory stimuli. Behav Brain Res 84:117-127.

Linster C, Hasselmo ME (1999) Behavioral responses to aliphatic aldehydes can be predicted from known electrophysiological responses of mitral cells in the olfactory bulb. Physiol Behav 66:497-502.

Linster C, Hasselmo ME (2001) Neuromodulation and the functional dynamics of piriform cortex. Chem Senses 26:585-594.
Linster C, Wyble BP, Hasselmo ME (1999) Electrical stimulation of the horizontal limb of the diagonal band of broca modulates population EPSPs in piriform cortex. J Neurophysiol 81:2737-2742.

Linster C, Garcia PA, Hasselmo ME, Baxter MG (2001a) Selective loss of cholinergic neurons projecting to the olfactory system increases perceptual generalization between similar, but not dissimilar, odorants. Behav Neurosci 115:826-833.

Linster C, Johnson BA, Yue E, Morse A, Xu Z, Hingco EE, Choi Y, Choi M, Messiha A, Leon M (2001b) Perceptual correlates of neural representations evoked by odorant enantiomers. J Neurosci 21:9837-9843.

Linster C, Johnson BA, Morse A, Yue E, Leon M (2002) Spontaneous versus reinforced olfactory discriminations. J Neurosci 22:6842-6845.

Mandairon N, Stack C, Kiselycznyk C, Linster C (2006a) Broad activation of the olfactory bulb produces long-lasting changes in odor perception. Proc Natl Acad Sci U S A 103:13543-13548.

Mandairon N, Ferretti CJ, Stack CM, Rubin DB, Cleland TA, Linster C (2006b) Cholinergic modulation in the olfactory bulb influences spontaneous olfactory discrimination in adult rats. Eur J Neurosci 24:3234-3244.

Mandairon N, Didier A, Linster C (2008a) Odor enrichment increases interneurons responsiveness in spatially defined regions of the olfactory bulb correlated with perception. Neurobiol Learn Mem 90:178-184.

Mandairon N, Peace S, Karnow A, Kim J, Ennis M, Linster C (2008b) Noradrenergic modulation in the olfactory bulb influences spontaneous and reward-motivated discrimination, but not the formation of habituation memory. Eur J Neurosci 27:1210-1219.

Meister M, Bonhoeffer T (2001) Tuning and topography in an odor map on the rat olfactory bulb. J Neurosci 21:1351-1360.

Metherate R, Weinberger NM (1990) Cholinergic modulation of responses to single tones produces tone-specific receptive field alterations in cat auditory cortex. Synapse 6:133-145.

Metherate R, Tremblay N, Dykes RW (1988) The effects of acetylcholine on response properties of cat somatosensory cortical neurons. J Neurophysiol 59:1231-1252.

Mori K, Nagao H, Yoshihara Y (1999) The olfactory bulb: coding and processing of odor molecule information. Science 286:711-715.

Pignatelli A, Belluzzi O (2008) Cholinergic modulation of dopaminergic neurons in the mouse olfactory bulb. Chem Senses 33:331-338.

Pressler RT, Inoue T, Strowbridge BW (2007) Muscarinic receptor activation modulates granule cell excitability and potentiates inhibition onto mitral cells in the rat olfactory bulb. J Neurosci 27:10969-10981.

Ravel N, Elaagouby A, Gervais R (1994) Scopolamine injection into the olfactory bulb impairs short-term olfactory memory in rats. Behav Neurosci 108:317-324.

Roberts MJ, Zinke W, Guo K, Robertson R, McDonald JS, Thiele A (2005) Acetylcholine dynamically controls spatial integration in marmoset primary visual cortex. J Neurophysiol 93:2062-2072.

Sarter M, Bruno JP (1997) Cognitive functions of cortical acetylcholine: toward a unifying hypothesis. Brain Res Brain Res Rev 23:28-46.

Sarter M, Hasselmo ME, Bruno JP, Givens B (2005) Unraveling the attentional functions of cortical cholinergic inputs: interactions between signal-driven and cognitive modulation of signal detection. Brain Res Brain Res Rev 48:98-111.

Shipley MT, Ennis M (1996) Functional organization of olfactory system. J Neurobiol 30:123-176.

Timofeeva E, Dufresne C, Sík A, Zhang ZW, Deschênes M (2005) Cholinergic modulation of vibrissal receptive fields in trigeminal nuclei. J Neurosci 25:9135-9143.

Weinberger NM (2003) The nucleus basalis and memory codes: auditory cortical plasticity and the induction of specific, associative behavioral memory. Neurobiol Learn Mem 80:268-284.

Wilson DA (2000) Comparison of odor receptive field plasticity in the rat olfactory bulb and anterior piriform cortex. J Neurophysiol 84:3036-3042.

Wilson DA (2001) Scopolamine enhances generalization between odor representations in rat olfactory cortex. Learn Mem 8:279-285.

Yokoi M, Mori K, Nakanishi S (1995) Refinement of odor molecule tuning by dendrodendritic synaptic inhibition in the olfactory bulb. Proc Natl Acad Sci U S A 92:3371-3375.

Yu AJ, Dayan P (2005) Uncertainty, neuromodulation, and attention. Neuron 46:681-692. 